\title{
HERITABLE COLON CANCER SYNDROMES: LONG-TERM B-LYMPHOCYTE SUSPENSION CULTURES DERIVED FROM COLONIC MUCOSAL BIOPSIES
}

\author{
by \\ B. SHANNON DANES
}

\begin{abstract}
Laboratory for Cell Biology, Department of Medicine, Cornell University Medical College, New York, USA
\end{abstract}

Keywords: Colon cancer genotypes, colonic mucosa biopsy, primary colon explant culture, B-lymphocyte suspension culture and long-term line

Human B-lymphocyte suspension cultures of known cancer genotypes have been established from colonic mucosal biopsies taken during colonoscopy. These lines were derived from primary biopsies from patients with an autosomal dominant colon cancer syndrome ( 3 FPC, I CFS, 1 HCC) who previously had a colonic adenocarcinoma surgically removed but had no evidence of clinical cancer at the time of biopsy. Immunological typing demonstrated that the cells of each line were of colonic and clonal origin. All have been characterized and samples frozen as reference source.

No lymphoid suspension cultures were established from similar cultures derived from 44 other patients with and 39 clinical normals without such a colon cancer syndrome.

Such B-lymphocyte suspension cultures should provide (a) a unique source of non-senescing normal cells with known cancer genotypes growing in suspension and (b) support the concept that there is an association between the presence of B-lymphocytes and cancer gene expression in the colon wall.

\section{INTRODUCTION}

The distribution of lymphocytes in the intestinal wall has been shown to be influenced by disorders of mucosal proliferation including adenomas, adenomatosis and adenocarcinomas. Although lymphocyte infiltration has been observed to be associated with such abnormal mucosa, its biological significance and relationship to carcinogenesis has not been established.

Observations have been limited to pathological descriptions, as there has been no in vitro cell model to study this phenomenon. The purpose of this paper is to report such a system. Long-term suspension cultures of B-lymphocytes have been established from colonoscopy biopsies taken from 5 patients with different autosomal dominant colon cancer syndromes who previously had a colonic adenocarcinoma surgically removed but had no evidence of clinical cancer at the time of the biopsy for culture was taken. No lymphoid suspension cultures were derived from similar cultures established from 44 other patients with and 39 without such a syndrome.

\footnotetext{
Abbreviations: CFS = Cancer Family Syndrome; EMEM = Eagle's minimum essential medium; FBS = fetal bovine serum; FPC = Familial Polyposis Coli; GS = Gardner Syndrome; HCC = Site-Specific Colon Cancer; sc $=$ subcutaneously
} 


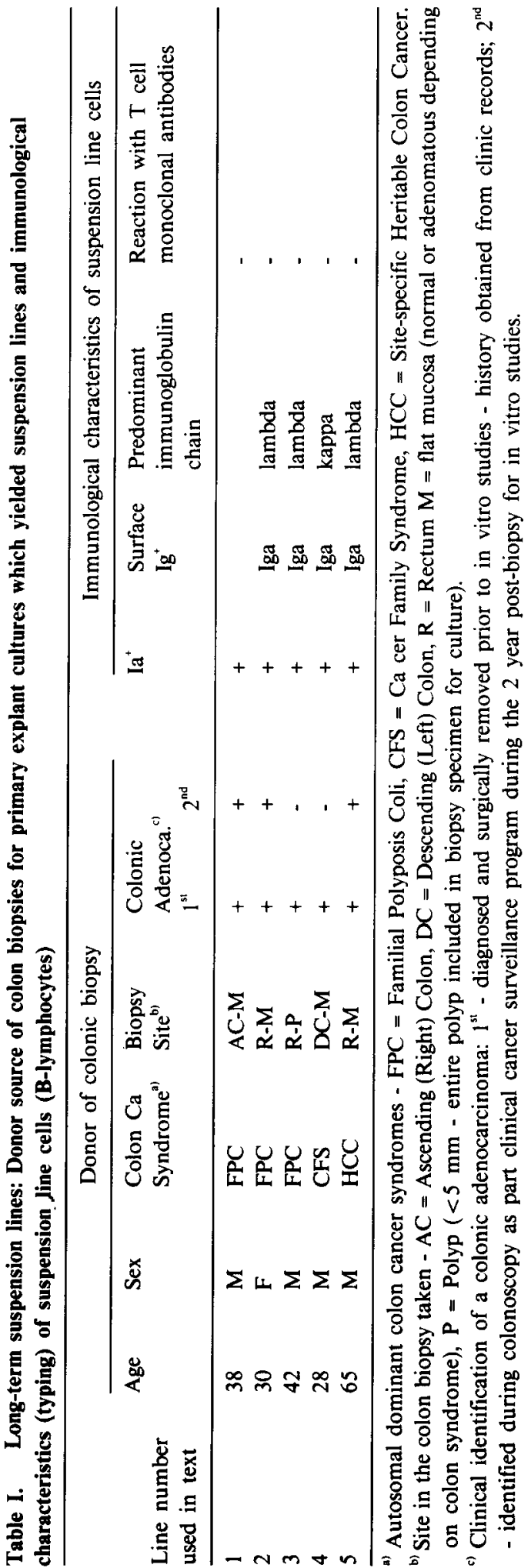

\section{MATERIALS AND METHODS}

\subsection{Study group}

Mucosal biopsies were obtained from 49 patients (20 female, 29 male, 21-65 years) with an autosomal dominant colon cancer syndrome without clinical evidence of colon cancer: syndromes with polyposis coli - 25 familial polyposis coli (FPC) and 10 Gardner syndrome (GS); syndromes without polyposis coli - 5 Cancer Family Syndrome (CFS) and 9 Site-Specific Colon Cancer (HCC). Colonic biopsis were also taken from 39 patients (age and sex matched with syndrome patients) without clinical colon cancer or a familial association (no first or second degree relative with colon cancer).

The biopsy $(1-2 \times 3 \mathrm{~mm})$ was obtained from each syndrome patient during a routine evaluation of colonic status. For the non-syndrome patient a specimen was taken during colonosocpic examination for evaluation of clinical symptoms. Either flat mucosa or an entire small polyp ( $\langle 5 \mathrm{~mm}$ ) was biopsied (Table I).

\subsection{Establishment of the primary mixed (epithelial-fibroblastic) cultures}

The biopsy was immediately placed in chilled culture medium (modified EMEM with 10\% (vol) FBS containing $50 \mu \mathrm{g}$ gentamicin and Fungizone/ml medium ( $\mathrm{pH} 7.4$ ) to be taken to the laboratory (Figure 1). Each biopsy was then cut into small pieces $(1 \times 1 \mathrm{~mm})$, placed in a culture tube containing the same medium, and kept at $4{ }^{\circ} \mathrm{C}$ for 12 hours. The mucosal pieces were then cut into smaller fragments and washed in fresh medium.

The culture vessel used was a double petri dish (a $60 / 15 \mathrm{~mm}$ dish in a $100 / 20 \mathrm{~mm}$ dish as isolation against external contamination). Four such fragments (explants) were placed in the inner dish containing approximately $1 \mathrm{ml}$ medium (EMEM with 25\% (vol) FBS without any antibiotics, $\mathrm{pH}$ 7.4). Optimal cellular migration from the explants was obtained when a 1 $\mathrm{ml} / 28 \mathrm{~cm}^{2}$ surface area was used to just cover the explants and floor of the culture dish. The culture vessel was then placed in an incubator having a humidified atmosphere of $5 \% \mathrm{CO}_{2}$ in air at $37^{\circ} \mathrm{C}$ and left undisturbed for 10 days. At that time each culture was examined micro- 


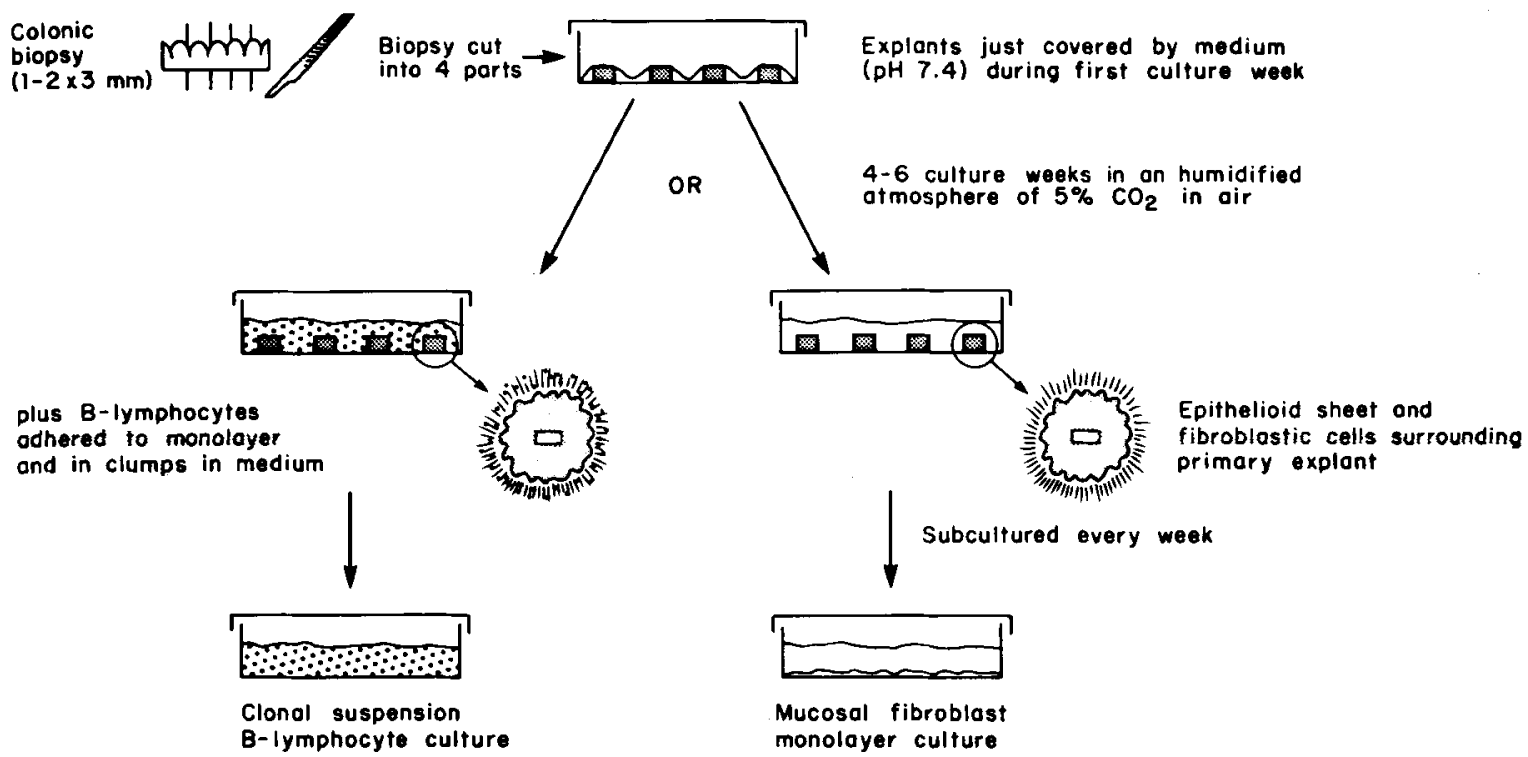

Figure 1. Diagrammatic outline of the methodology used in the establishment of long-term B-lymphocyte suspension lines from primary explant cultures derived from colonic mucosal biopsies taken during colonoscopic examinations of patients with autosomal dominant colon cancer syndromes (FPC, CFS and HCC).

scopically for cellular migration. Explants that showed no cellular migration were removed. Three ml of medium was added to the culture with care being taken not to loosen the explants and The medium was completely changed once a week for the remaining culture period of the primary culture.

During the culture time (usually 4 culture weeks) for cells to migrate from the explants and multiply to cover the culture floor, round cells were observed attached to cell surfaces composing the monolayer or in clumps in the medium. When such cells were noted, only half the medium was changed each week being careful not to dislodge the adhered cells or lose the suspension cells. When there appeared to be a sufficient suspension population, these cells were transferred to a new culture vessel. Half the medium was discarded; a Pasteur pipette was used to dislodge the cells adhered to the monolayer as well as to break up the cell clumps in the medium. This cell suspension was then introduced into a new culture vessel which was then placed in a humidified $\mathrm{CO}_{2}$ incubator for subsequent growth. In this and subsequent subcultures the "supporting cell", the fibroblast monolayer, was no longer present. After the suspension cells were transferred, the fibroblast monolayer was subcultured into 2 culture vessels as previously described in detail (1) and kept as a separate cell line.

Samples of both the cell suspension and monolayer cultures were stored in liquid nitrogen after the second subculture (approximately 2 months after the initial explant culture was established).

During subsequent continuous culture periods (at present over 38 months), each suspension culture was subcultured weekly by discarding the major part of the culture medium and transferring $1 \mathrm{ml}$ of the remaining medium containing suspension cells to a new culture vessel containing $4 \mathrm{ml}$ of fresh medium. 


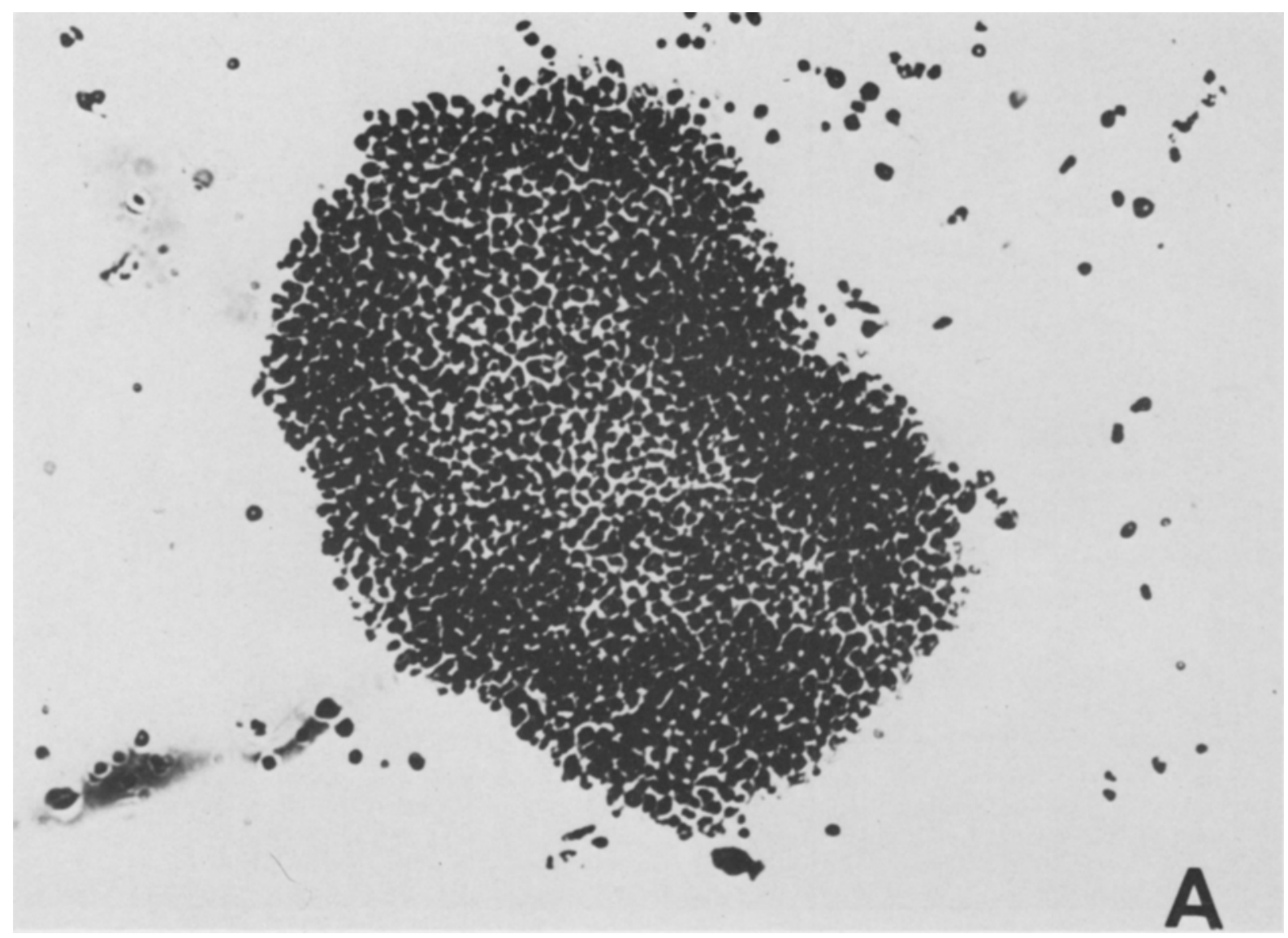

\subsection{Characterization of suspension cells}

Morphology was examined under light, phase and transmission microscopies. Growth kinetics were studied by methods previously described in detail (1). Every month after establishment of the suspension lines, chromosome preparations were made, stained in acetic orcein, and examined for variations from diploidy in chromosome complement. Growth of cells in the nude mouse was determined for evidence of tumorigenicity (3) and their secretory immunoglobulins characterized (5).

\section{RESULTS}

3.1. Growth pattern in primary explant cultures

Sheets of epithelium were observed in the migration zone around each explant during the first culture week. Migration of fibroblasts from an explant started in the second culture week and continued as long as the explant was present. In the majority ( 78 of 83 cultures) of colonic mucosa explant cultures initiated, these were the only two identifiable cell types.

In all the primary cultures derived from colon biopsies from 5 patients a third cell type appeared between the fourth and sixth culture week. This cell type was distinctly different from the other two types - a round cell which either grew in clumps (aggregates) in the culture medium (Figure 2) or adhered as single cells and groups to the cell surface of the monolayer.

\subsection{Culture after establishment of suspension lines}

The suspension lines had to be subcultured weekly to maintain a cell density in the range 


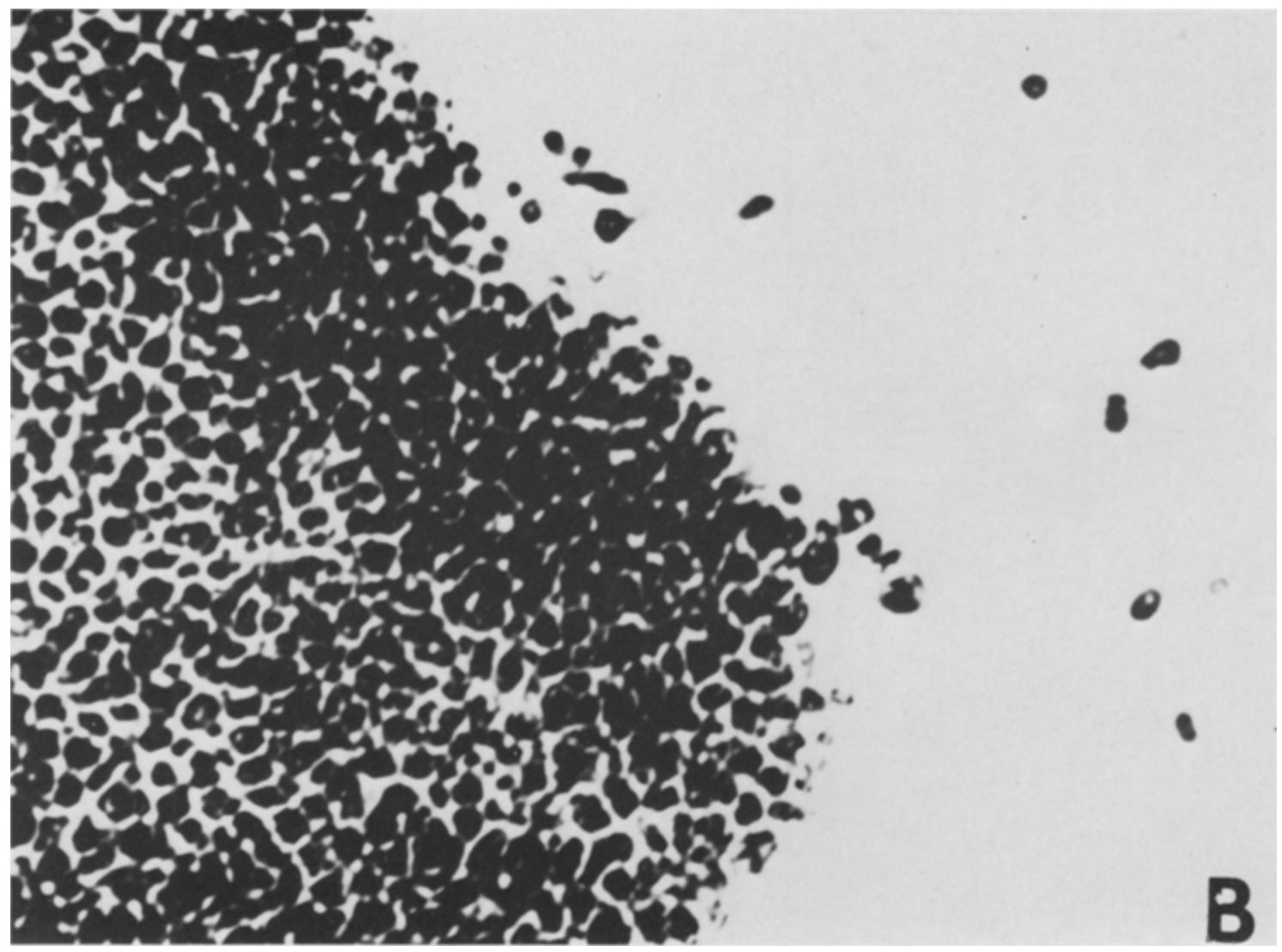

Figure 2. Light micrograph of living B-lymphocytes (single and aggregates) growing as a suspension line derived from a colonic mucosal biopsy from an FPC patient (no. 2, Table I). Line had been in continuous culture for over 3 years when photographed. (A) Cell aggregate. (B) Portion of same aggregate. Original magnification of (A) 100 and (B) 200.

of $4-40 \times 10^{3}$ cells per $\mathrm{cm}^{2}$ floor area of the culture vessel. Under this culture regimen, the lines have been maintained in continuous culture (Table I) maintaining the characteristics described below.

\subsection{Characteristics of suspension cell}

As the suspension cells in all cultures irrespective of donor source had the same features, it was assumed that in a primary explant culture a cell with the ability to proliferate under the in vitro culture conditions established was selected for.

\subsubsection{Morphology}

The suspension cell had the morphological features of a lymphocyte - a large nucleus containing a prominent single nucleolus, scant lightly staining cytoplasm containing ribosomes and rough endoplasmic reticulum, and a high nuclear/cytoplasmic ratio (Figure 3 ).

\subsubsection{Growth kinetics}

During logarithmic growth (Figure 4) the average generation time for all suspension lines was $14 \pm 7$ hours. The lines derived from FPC patients had generation times of approximately 15 hours \pm 7 hours (cell line no. $1-17$ hours, no. 2 - 17 hours, no. $3-12$ hours); the line derived from the HCC patient studied had a generation time of 8 hours. During continuous culture the generation times did not show any significant or consistent change (i.e. the generation time of no. 2 during logarithmic growth 


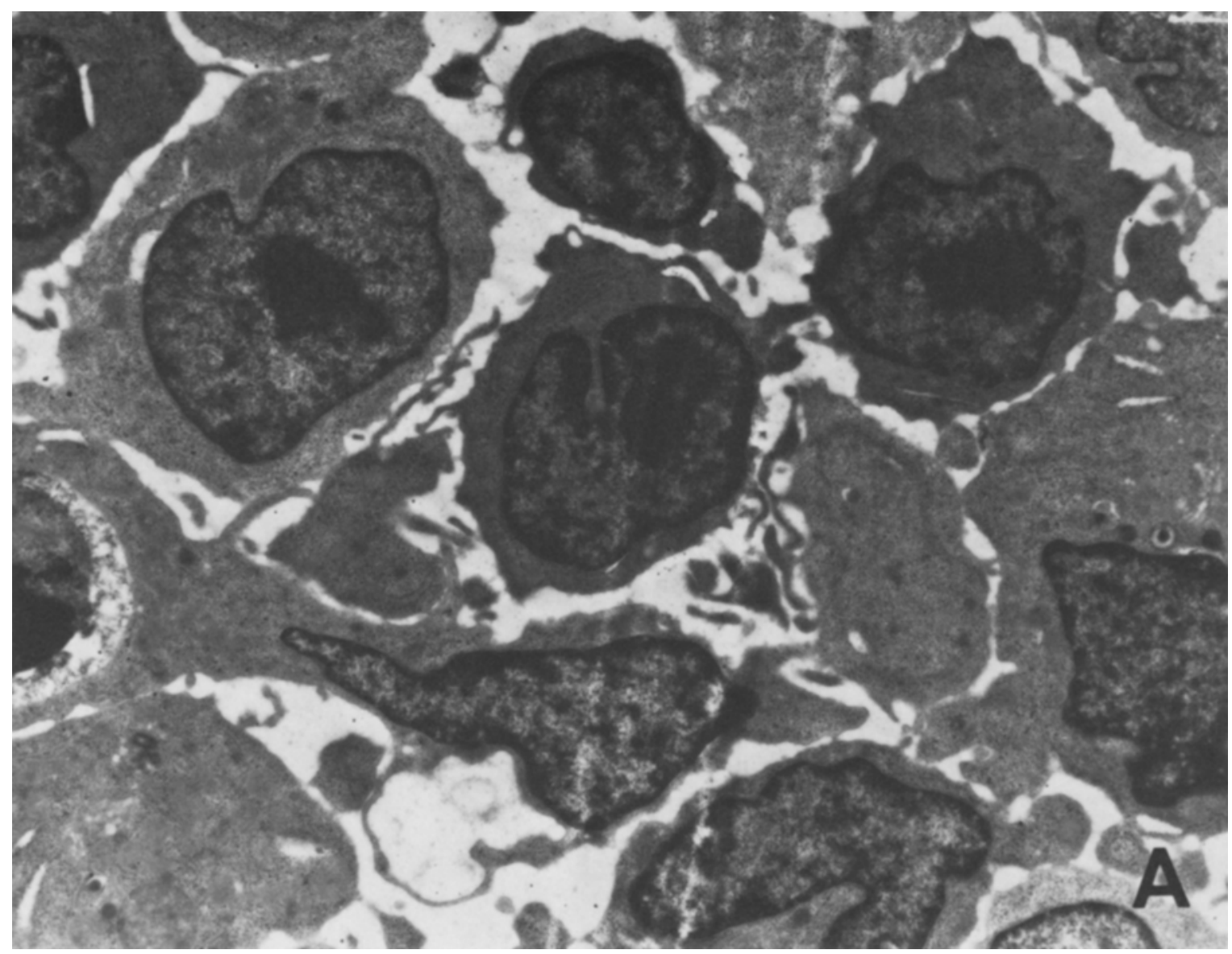

ranged from $16-17$ hours over a 3 year continuous culture period). Whereas the generation times of colonic fibroblasts derived from the same primary colon cultures of no. 2 averaged 25 hours \pm 4 hours during the second culture month (5th subculture), had ceased to grow and been discarded by the seventh culture month (20 subcultures) (1).

\subsubsection{Chromosome complements}

The karyotypes were diploid ( $46 \mathrm{XX}$ or XY) without obvious chromosome aberrations. The chromosome frequency distribution shortly after the establishment of each suspension line (1 month) was $99 \%$ diploid and $1 \%$ hyperdiploid. After 30 continuous culture months, there had been little change in this distribution (98-100\% diploid and 0-2\% hyperdiploid).

\subsubsection{Tumorigenicity}

Tumorigenicity was not observed because none of the samples from each cell line inoculated sc into athymic nude mice produced tumors in a 6-month period.

\subsubsection{Immunological typing}

In all 5 long-term suspension cultures all the cells were demonstrated to be B-lymphocytes as none showed a reaction with $\mathrm{T}$ cell monoclonal antibodies (Table I). Cell surface immunoglobulin was universally A (Iga). The predominant immunoglobulin chain was lambda for 3 (2 FPC and $1 \mathrm{HCC}$ ) and kappa for 1 (CFS) indicating the clonal origin of these individual suspension lines. 


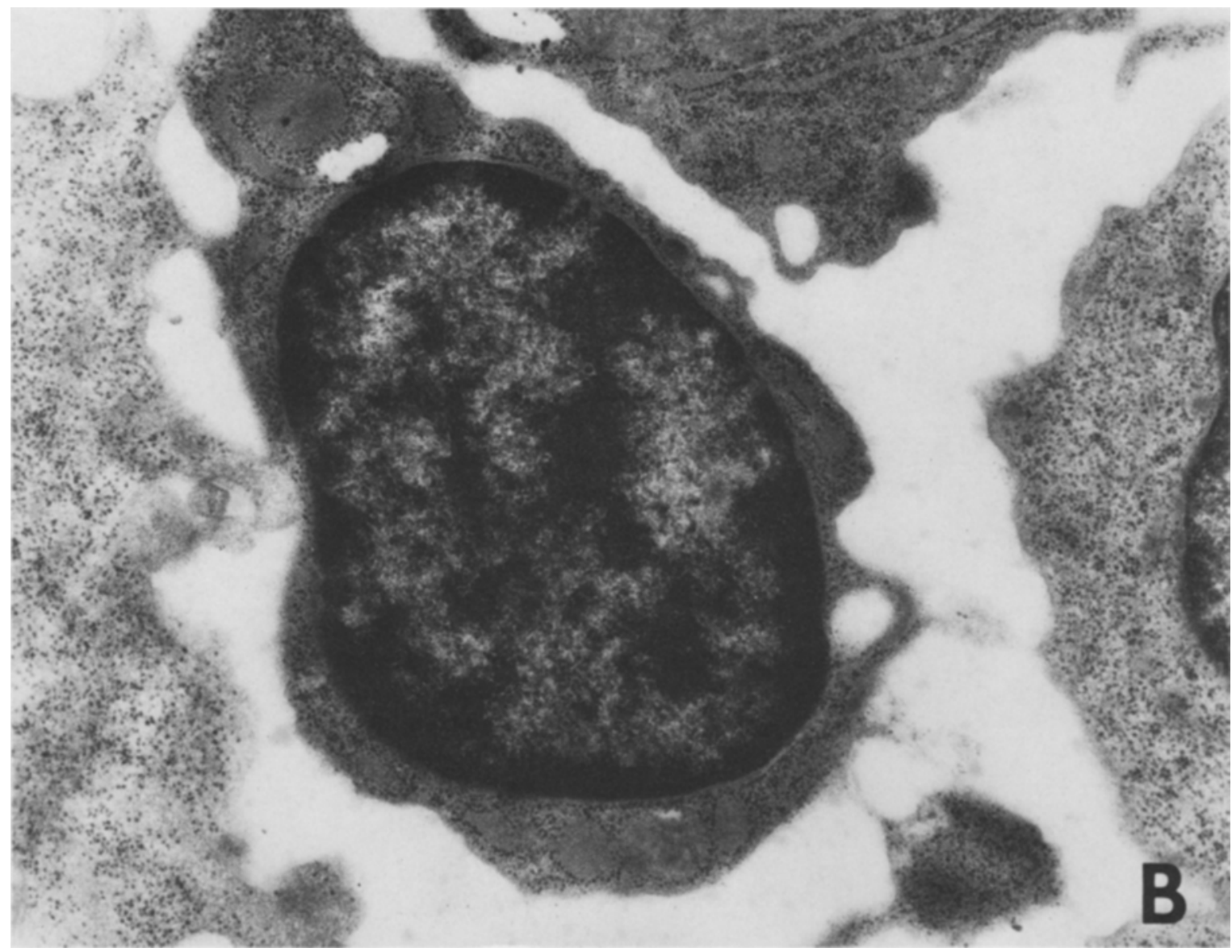

Figure 3. Electron micrographs of B-lymphocytes from suspension line no. 2 (see legend of Figure 2 and Table I) illustrating the morphological features of the B-lymphocyte comprising the established suspension lines described in text. (A) cell aggregates, (B) single cell. Original magnification of (A) 10,000 and (B) 67,000.

\section{DISCUSSION}

The B-lymphocyte was the cell type comprising the suspension population in all 5 lines derived from colonic mucosa biopsies. Their colonic origin was based on the demonstration of (a) B-type of lymphocyte and (b) immunoglobulin $\mathrm{A}$ (Iga) as the cell surface antigen, the species associated with the mucous membranes having continuity with the external environment (5). The cells appeared to be lymphocytes and not lymphoblasts as (a) their morphological and staining characteristics were consistent with the former and (b) their nontumorigenicity in the nude mouse.

Only 1 difference in growth of the 5 lines was observed (Figure 4). The generation times dur- ing logarithmic growth for the 3 FPC lines were similar (approximately 15 hours); that of the $\mathrm{HCC}$ was shorter (approximately 8 hours). The biological reason for such a difference in in vitro generation times of colonic B-lymphocytes with different cancer genotypes was not clarified; however, the proliferative pattern in the colonic mucosa biopsies was dissimilar (adenomatous in FPC and normal in $\mathrm{HCC}$ ).

Characterization of the B-lymphocyte of these suspension lines established the following observations: (a) Abnormal proliferation in the colonic mucosa biopsied (i.e. adenomatosis) was not a requirement for the establishment of a lymphoid suspension culture from the biopsy specimen. Suspension cultures were initiated 
from both biopsies of adenomatous (FPC - no. 1 - no. 3 lines from FPC patients) and flat, normal mucosa (CFS - no. 4, HCC - no. 5). (b) Suspension lines arose only in primary explant cultures derived from affecteds with a heritable colon cancer syndrome. None of the cultures established from colon biopsies from 39 patients without colon pathology related to a cancer syndrome yielded a B-lymphocyte suspension line. (c) An unknown influence in the

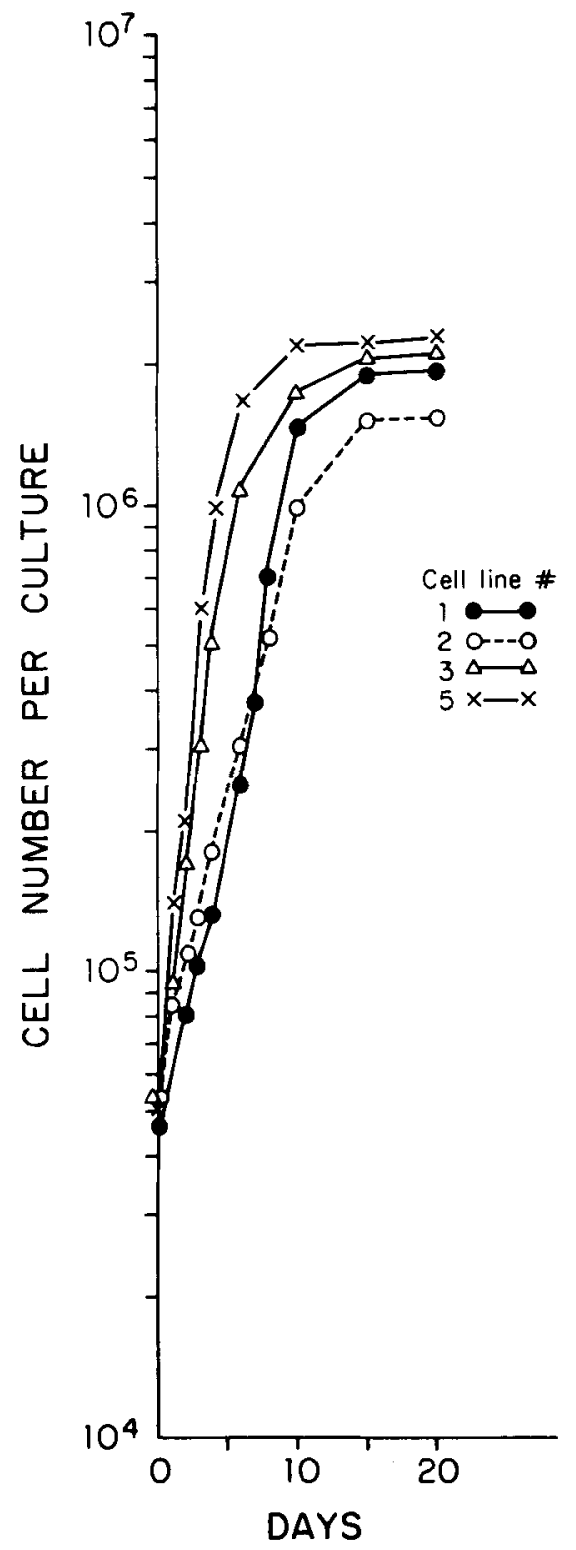

colonic mucosa biopsied necessarily is the cruical factor in the subsequent in vitro development of such a suspension line. Only a minority, $10 \%(5 / 49)$ of the patients with a heritable colon cancer syndrome gave colonic biopsies which on culture yielded a suspension line. (d) The development of the suspension line was not associated with clinical colon cancer including in situ cancer at the time of the biopsy. (e) Unlike the lymphoid suspension cultures established from peripheral blood (4), these B-lymphocytes have not been stimulated by a mitogen.

Often the increased presence of B-lymphocytes in the colonic mucosa showing abnormal mucosal proliferation (i.e. adenomatosis) has been noted but its biological significance or relationship to carcinogenesis in the colon has not been established. Variation in the incidence of gut-infection with its associated lymphocyte infiltration into the colonic mucosa has been suggested to be related to known geographical variation in the incidence of colon cancer (6). It has been proposed that a study of the intraepithelial lymphocytes in a high and a low-incidence country could be informative (2). The present in vitro study did not add data concerning these speculations. However, the data did suggest that there had been a change in the colonic B-lymphocyte with colon cancer genotypes from certain patients so that an au-

Figure 4. Growth of B-lymphocyte suspension lines with known colon cancer syndrome genotypes derived from primary explant cultures of colonic mucosa. Mucosal biopsies obtained from patients with an autosomal dominant colon cancer syndrome without clinical evidence of colon cancer at the time the biopsy was taken during routine colonoscopy for cancer surveillance. Donor source of biopsy:nos. 1, 2 and 3 - FPC, no. 5 - HCC (see Table I for additional data).

Suspension lines had grown in continuous culture for 18 months prior to the growth studies shown in figure. Cell suspension samples $\left(5 \times 10^{4}\right.$ cells) were plated into a set of $60 / 15 \mathrm{~mm}$ petri dishes containing $3 \mathrm{ml}$ medium (EMEM with 25\% (vol) FBS) and grown in a humidified atmosphere of $5 \% \mathrm{CO}_{2}$ in air at 37 ${ }^{\circ} \mathrm{C}$ (pH 7.4). One $\mathrm{ml}$ of fresh medium was added on the 7th and 14th culture day. Cell counts were done on duplicate cultures throughout a 20 day culture period. 
tonomous suspension population of clonal origin with an infinite life span could arise from a single colonic B-lymphocyte.

The 5 patients whose primary explant cultures yielded B-lymphocyte suspension cultures have received clinical evaluations at regular intervals. In the 2 years after their biopsy was taken for in vitro studies, 3 (no. 1, no. 2 and no. 5) each had another colonic adenocarcinoma (no. 1 at the hepatic flexure, no. 2 at the splenic flexure and no. 5 in the transverse portion of the colon). All the biopsies from these 3 patients had been taken at least $20 \mathrm{~cm}$ from the site of the second carcinoma. The other 2 patients had no new lesions detected by colonoscopy in this 2 year post-biopsy period.

Although it has been recognized that all patients with such colon cancer syndromes should receive periodic clinical surveillance, the present data suggested that a patient whose primary colonic explant culture yields a B-lymphocyte suspension line should perhaps receive even closer evaluation. This suggestion was based on 2 observations reported in this paper: (a) $10 \%$ $(5 / 49)$ of the affecteds with such colon cancer syndromes studied produced such suspension lines and (b) 3 of these 5 had a second colonic adenocarcinoma in the 2 years post-biopsy period. Further in vitro research on colonic biopsies from such patients may well demonstrate that the incidence of B-lymphocyte suspension line from a primary colonic explant culture has prognostic value for screening for the clinical occurrence of an adenocarcinoma in the colon.

The data presented in this paper suggested that in vitro studies may now be contemplated through the availability of B-lymphocyte suspension lines of known cancer genotypes established from colonic mucosal biopsies.

\section{ACKNOWLEDGEMENTS}

The author wishes to thank Dr. BENJAMIN KOZINER for arranging for the immunological typing of the suspension cell lines to be done in the Surface Marker Laboratory, Memorial Sloan-Kettering Cancer Center and for his interpretation of this data. This research was made possible through a grant (CA 15973) from the National Large Bowel Cancer Project, Division of Cancer Research Resources and Centers, National Cancer Institute and a NATO grant (no. 2013).

\section{REFERENCES}

1. DANES, B.S.: Epithelial line from normal human colon mucosa. J. Natl. Cancer Inst. 69, 1271-1276 (1982)

2. DE Sousa. M. \& R.A. Good: T- and B-cell populations in gut and gut-associated lymphoid organs: arrangement, migration, and function. In: Gastrointestinal Tract Cancer, Lipkin and Good, eds., Plenum Publish. pp. 29-47 (1978)

3. Friedman, E.A. P.J. Higgins. M. Lipkin, H. ShiNYA \& A.M. GELB: Tissue culture of human epithelial cells from benign colonic tumors. In Vitro 17, 632-644 (1981)

4. Moore. G.E., E. Ito, K. Ulrich \& A.A. SandBERG: Culture of human leukemia cells. Cancer 19, 713-723 (1966)

5. TOMasi, T.B.JR.: Secretory immunoglobulins. N. Engl. J. Med. 287, 500-506 (1972)

6. Wynder. E.L.. B.S. Reddy, G.D. MCCoy, J.H. Weisburger \& G.M. Willians: Diet and gastrointestinal cancer. Clin Gastroenterol. 5, 463.482 (1976) 